\title{
Cooperative Coverage using Receding Horizon Control
}

\author{
Ali Ahmadzadeh, Ali Jadbabaie, Vijay Kumar and George J. Pappas
}

\begin{abstract}
We present a path planning algorithm for cooperative surveillance using a set of heterogeneous unmanned vehicles. The paper describes our overall framework and algorithm for the computation of trajectories that maximize spatio-temporal coverage while satisfying hard constraints such as collision avoidance and specifications on initial and final positions. An Integer Programming (IP)-based strategy for successfully operating within these constraints is developed. IP is applied over a receding planning horizon with terminal cost to reduce the computational effort of the planner and to incorporate feedback. Simulation and results are presented to demonstrate the efficacy of the proposed approach.
\end{abstract}

\section{INTRODUCTION}

$\mathbf{T}$ HE use of Unmanned Aerial Vehicles (UAVs) equipped with cameras for surveillance is a natural but challenging application of robotic technology. Unlike ground robots, aerial platforms have many more operating constraints and their dynamic response typically dictates the nature of their role in such an application. [1]-[6].

Consider the problem of designing a path planner for a set of unmanned vehicles with the following scenario. The planner receives a request for a plan for a specified subset of vehicles under its control. The planner also receives an entry and exit state for each vehicle (3-D location, velocity vector, and time of arrival). It also receives additional information such as obstacle or threat locations, no-fly zones, at sensor availability. The task of the planner is the computation of trajectories that maximize spatio-temporal coverage while satisfying hard constraints such as collision avoidance and specifications on initial and final positions.

This paper address the cooperative motion-planning problem for a group of heterogeneous vehicles which have to surveil and generate mosaic maps of their operations area while respecting boundary conditions. Each vehicle is modeled as a nonholonomic point mass moving on a twodimensional (2-D) plane at a constant speed with a minimum turning radius. This model is also known as Dubins car in the literature [7]. There is extensive research on multi-UAV task scheduling and planning which doesn't address the coverage problem. For a review the interested reader can refer to [11], [12]. One approach to the coverage problem is based on cellular decomposition. When using a cellular decomposition either each cell is covered when the vehicle crosses it or the

\footnotetext{
$\dagger$ This research is supported in parts by the following grants: Army Research Office DURIP Grant W911NF-04-1-0148, ARO-MURI W911NF05-1-0381, ONR/YIP N00014-04-1-0467, ONR N00014-06-1-0436, and NSF-ECS-0347285.

Authors are with GRASP Laboratory, University of Pennsylvania, Philadelphia, PA 19104,USA

\{aliahmad, jadbabai, kumar, pappasg\} @grasp.cis.upenn.edu
}

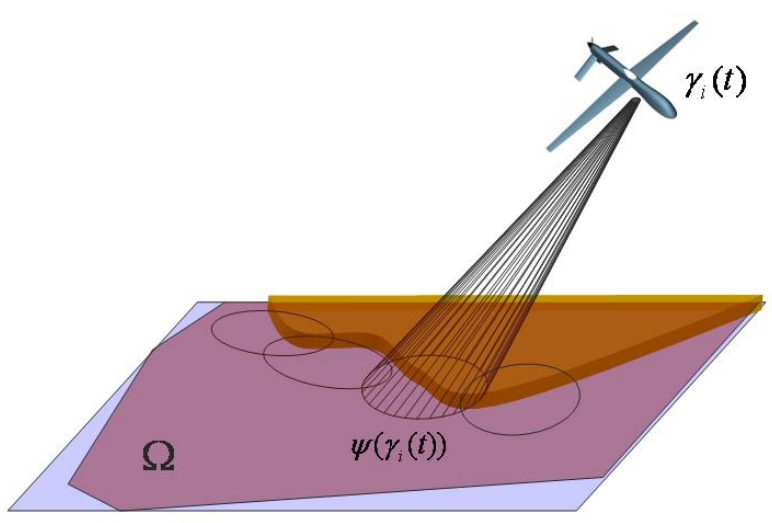

Fig. 1. Sensor footprint map of onboard sensors

vehicle has to perform some specific motion pattern (usually a boustrophedon motion) in order to cover the cell [13]. This method mainly focuses on the single robot coverage and nonholonomic constraints are not considered.

Most of prior work in the area of coverage concentrated on sensor network problem [14]-[17].The cooperation of multiple sensors is often achieved by choosing different sensors (or sensor modes) for different tasks (targets) at different times and motion planning is not involved in these problems. Cortes et al. [18] studied the multi-sensor localization problem in a polygonal environment and developed a gradient-descent algorithm for optimal coverage and sensing policies. Each sensor agent is expected to converge to its optimal location and stay there. Enrigh et al. [19] considered the problem of visiting stochastically-generated targets in a planar bounded region with the objective of minimization of the expected waiting time between the appearance of a target, and the time it is visited. In [21], [22] the coupling between flight path and the camera field of view is studied and an Integer Programming(IP)-based algorithm for time-critical cooperative surveillance using a set of UAVs is introduced.

\section{Problem Definition}

The mathematical formulation of the problem for the $T I C^{3}$ planner include the following components.

1. $N$ heterogeneous autonomous vehicles: These vehicles work at fixed altitudes and might have conflict when they are on the same altitude. Vehicle $i$ has constant forward velocity $v_{i}$ and minimum turning radius $\rho_{i}$ and a planar trajectory as $\gamma_{i}(t)=\left(x_{i}(t), y_{i}(t)\right)$. 


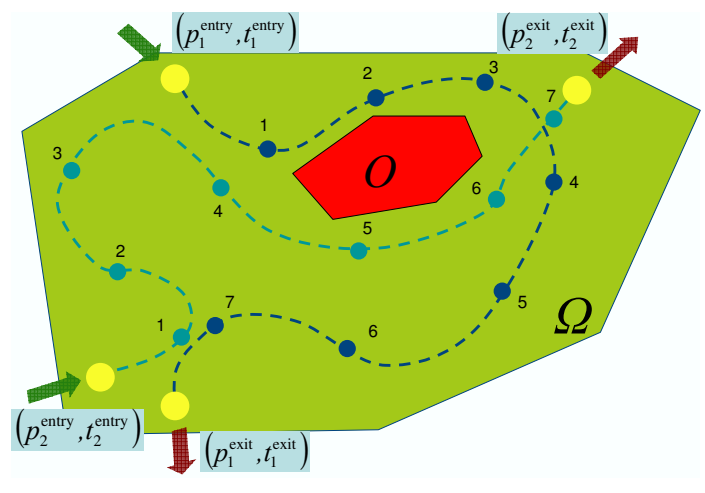

Fig. 2. An example with two vehicles

2. Boundary conditions, $\left\{\gamma_{i}^{\text {entry }}, t_{i}^{\text {entry }}\right\}$ and $\left\{\gamma_{i}^{\text {exit }}, t_{i}^{\text {exit }}\right\}$, for $i=1,2, \cdots, N$ : These conditions mean that the vehicle $i$ starts the task at position $\gamma_{i}^{\text {entry }}$ at time $\gamma_{i}^{\text {entry }}$, and terminates the task at position $\gamma_{i}^{\text {exit }}$ at time $\gamma_{i}^{\text {exit }}$. We use $T_{i}=t_{i}^{\text {exit }}-t_{i}^{\text {entry }}$ to denote the time horizon of vehicle $i$.

3. Coverage area, $\Omega \subset \mathfrak{R}^{2}$ : It is a closed and bounded subset of $\mathfrak{R}^{2}$.

4. Non-fly or non-sail zones, $O \subset \mathfrak{R}^{2}$ : They are closed and bounded subsets of $\mathfrak{R}^{2}$, which are characterized as the union of a finite number of polygonal regions.

5. Sensor footprint mapping, $\Psi$ : Each vehicle is equipped with a device, which will sense the situations on the ground. The sensing footprint of the device is characterized by $\Psi_{i}: \gamma_{i} \rightarrow 2^{R^{2}}$. Fig. 1

An exact solution for this problem is trajectories $\left\{\gamma_{1}^{*}(t), \cdots, \gamma_{N}^{*}(t)\right\}$ for $N$ vehicles such that

$$
\left\{\gamma_{1}^{*}(t), \cdots, \gamma_{N}^{*}(t)\right\}=\arg \max _{\left\{\gamma_{1}(t), \cdots, \gamma_{N}(t)\right\}} \bigcup_{t}\left(\Psi_{i}\left(\gamma_{i}(t)\right) \cap \Omega\right)
$$

while avoiding non-fly or non-sail zones $O \cap\left\{\gamma_{i}^{*}(t)\right\}=\emptyset$, satisfying boundary conditions

$$
\gamma_{i}^{*}\left(t_{i}^{\text {entry }}\right)=\gamma_{i}^{\text {entry }}, \gamma_{i}^{*}\left(\gamma_{i}^{\text {exit }}\right)=\gamma_{i}^{\text {exit }}
$$

and the minimum turning radius constraints, for $i=$ $1,2, \cdots, N$. To apply these solutions on practical vehicles, these admissible trajectories will be converted into a sequence of way points for each vehicles. An example of the problem with two vehicles is shown in Fig. 2, in which entry and exit states and time are marked as large dots, dashed lines represent the solution trajectories, and small dots represent way points on the solution trajectories. Following these trajectories, vehicles will achieve maximal coverage while avoiding non-fly or non-sail zones.

If boundary conditions (16) are replaced by

$$
\gamma_{i}^{*}\left(t_{i}^{\text {entry }}\right)=\gamma_{i}^{\text {entry }},\left\|\gamma_{i}^{*}\left(t_{i}^{o}\right)-\gamma_{i}^{\text {exit }}\right\| \leq \alpha_{i}^{d},\left|t_{i}^{o}-t_{i}^{\text {exit }}\right| \leq \alpha_{i}^{t},
$$

then the solution is called an approximate solution with spatial tolerance $\alpha^{d}=\left[\alpha_{1}^{d}, \alpha_{2}^{d}, \cdots, \alpha_{N}^{d}\right]$ and time tolerance $\alpha^{t}=\left[\alpha_{1}^{t}, \alpha_{2}^{t}, \cdots, \alpha_{N}^{t}\right]$.

Throughout the paper we use the following notations. Consider an n-tuple $\alpha=\left(\alpha_{1}, \ldots, \alpha_{n}\right)$ of nonnegative integers, then we define the sum as $[\alpha]=\sum \alpha_{i}$ and $\frac{\partial^{\alpha}}{\partial x^{\alpha}}=\frac{\partial^{[\alpha]}}{\partial x_{1}^{\alpha_{1}} \ldots \partial x_{n}^{\alpha_{n}}}$.
Let $f: \Re^{n} \rightarrow \mathfrak{R}$. We say that $f$ is class $C^{k}$ for nonnegative integer $k$, if the partial derivatives $\frac{\partial^{\alpha}}{\partial x^{\alpha}}$ exist and are continuous for $[\alpha] \leq k$. If $f: \mathfrak{R}^{n} \rightarrow \mathfrak{R}^{m}$, then $f$ is class $C^{k}$ if each of the component functions $f_{i}$ is class $C^{k}$. Also $\|$.$\| denote$ Euclidian norm.

For the set $V$, measure $\mu(V)$ is defined as:

$$
\mu(V)=\int_{V} \chi_{v}(x) d x
$$

where $\chi_{v}(x)$ is indicator function defined as:

$$
\chi_{v}(x)= \begin{cases}1 & x \in V \\ 0 & x \notin V\end{cases}
$$

For the set $V \subset \mathfrak{R}^{2}$, let $M(V)$ be the mass center of the inertia as:

$$
M(V)=\int_{V} x d x
$$

A planar path or trajectory is a function $\gamma:\left[t_{0}, t_{1}\right] \rightarrow \mathfrak{R}^{2}$ as $\gamma_{i}(t)=\left(x_{i}(t), y_{i}(t)\right)$. Signed curvature of the path $\gamma(t)$ is $\kappa(t)$ and is defined as:

$$
\kappa(t)=\left(x(t)^{\prime} y(t)^{\prime \prime}-x(t)^{\prime \prime} y(t)^{\prime}\right) /\left\|\gamma^{\prime}(t)\right\|^{3}
$$

A planar trajectory $\gamma_{i}(t)$ is called flyable by vehicle $u_{i}$ if it is class $C^{2}$ and the absolute value of the signed curvature of the trajectory is always less than $1 / \rho_{i}$ where $\rho_{i}$ is the minimum radius of a flyable circle by UAV $u_{i}$. Since the trajectory $\gamma_{i}(t)$ of UAV $u_{i}$ is a planar curve with constant speed $v_{i}$, signed curvature function $\kappa_{i}(t)$ and initial conditions $\gamma_{i}\left(t_{0}\right)$ and $\theta_{i}\left(t_{0}\right)$ are sufficient to fully specify trajectory $\gamma_{i}(t)$ as described in the following theorem adapted from [20].

Theorem 1: Let $\kappa:\left[t_{0}, t_{0}+\tau\right] \rightarrow[-1 / \rho, 1 / \rho]$ be a piecewise continuous curvature function for the planar trajectory $\gamma(t)$ with given initial conditions $\gamma\left(t_{0}\right)=\left(x\left(t_{0}\right), y\left(t_{0}\right)\right), \theta\left(t_{0}\right)$ and constant speed $\left\|\gamma_{i}^{\prime}(t)\right\|=v_{i}$. Then the parameterized trajectory $\gamma:\left[t_{0}, t_{0}+\tau\right] \rightarrow \mathfrak{R}^{2}$ can be written as:

$$
\gamma(t)=(x(t), y(t))
$$

where

$$
\begin{aligned}
& x(t)=v \int_{t_{0}}^{t} \cos (\theta(\zeta)) d \zeta+x\left(t_{0}\right) \\
& y(t)=v \int_{t_{0}}^{t} \sin (\theta(\zeta)) d \zeta+y\left(t_{0}\right)
\end{aligned}
$$

and

$$
\theta(\zeta)=\int_{t_{0}}^{\zeta} \kappa(\xi) d \xi+\theta\left(t_{0}\right)
$$

Also $\gamma(t)$ is a class $C^{2}$ and flyable trajectory for a UAV with constant speed $v$ and initial conditions $\gamma\left(t_{0}\right)$ and $\theta\left(t_{0}\right)$ with minimum turning radius $\rho$.

This theorem enables us to change the search space from flyable planar trajectories $\gamma_{i}(t) i=1, . ., N$ to bounded scalar functions $\kappa_{i}(t) i=1, . ., N$.

Therefore we can restate our objective as generating curvature functions $\left\{\kappa_{i}(t)|| \kappa_{i}(t) \mid \leq 1 / \rho_{i}, i=1,2, \cdots, N\right\}$ for all UAVs in order to get maximum coverage while satisfying hard constraints such as collision avoidance and specifications on initial and final positions. 
Assume that $\theta_{i}(t)$ is the heading of the vehicle $i$ with respect to the $y=0$ axis. For each vehicle $i$ at given time $t$, the position of vehicle $\gamma_{i}(t)$ and the heading of the vehicle $\theta_{i}(t)$ are sufficient to uniquely specify the field of view. The heading of each vehicle $\theta_{i}(t)$ can be written as:

$$
\theta_{i}(t)=\arctan \left(y_{i}^{\prime}(t) / x_{i}^{\prime}(t)\right)
$$

Given any time instant $t_{0}$, the values of $\gamma_{i}\left(t_{0}\right)$ and $\gamma_{i}^{\prime}\left(t_{0}\right)$ uniquely specify the field of view at time $t_{0}$. Based on this we define the state of configuration of the $\mathrm{N}$-vehicle system as follows.

Definition 1 (State of the system): The state of N-vehicle system with given trajectories $\left\{\gamma_{1}, \ldots, \gamma_{N}\right\}$ at any time $t$ is defined by a $N \times 4$ matrix as:

$$
s(t)=\left(\begin{array}{cc}
\gamma_{1}(t) & \gamma_{1}^{\prime}(t) \\
\vdots & \vdots \\
\gamma_{N}(t) & \gamma_{N}^{\prime}(t)
\end{array}\right)=\left(\begin{array}{c}
s_{1}(t) \\
\vdots \\
s_{N}(t)
\end{array}\right)
$$

Definition 2 (Coverage map $\Psi$ ): At any time $t$ coverage map is mapping from the configuration space to the set $\Omega$ as follows:

$$
\Psi(s(t))=\bigcup_{i=1}^{N} \psi_{i}\left(s_{i}(t)\right) \bigcap \Omega
$$

Note that $\Psi(s(t))$ is actually a snapshot of the coverage of $\Omega$ at time $t$.

Now we can define an alternative way coverage problem as follows:

$$
\left\{\kappa_{1}^{*}(t), \cdots, \kappa_{N}^{*}(t)\right\}=\arg \max _{\left\{\kappa_{1}(t), \cdots, \kappa_{N}(t)\right\}} \bigcup_{t}\left(\Psi_{i}\left(s_{i}(t)\right) \cap \Omega\right)
$$

while avoiding non-fly or non-sail zones $O \cap\left\{\gamma_{i}^{*}(t)\right\}=\emptyset$, satisfying boundary conditions

$$
\gamma_{i}^{*}\left(t_{i}^{\text {entry }}\right)=\gamma_{i}^{\text {entry }}, \gamma_{i}^{*}\left(\gamma_{i}^{\text {exit }}\right)=\gamma_{i}^{\text {exit }}
$$

The objective of the coverage problem is to find admissible curvature functions $\kappa_{i}(t) i=1 \ldots N$ to get maximum coverage of the region $\Omega$ while satisfying constraints such as collision avoidance and initial and final positions.

Mathematically, the coverage problem is an infinitedimensional non-convex optimization problem because its search space of all admissible trajectories of the vehicles is infinite dimensional, and coverage area and non-fly or nonsail zones might induce non-convex constraints. Computing an exact solution requires a finite and exact representation of the search space. Because such a representation is not available, we will discretize the problem in the next session to obtain an approximate discrete representation.

The complexity of the approximate discrete representation is still quite high. Exhaustively search will return the optimal solution in the representation, but not be able to satisfy the given time budget. We will use receding horizon algorithms to find suboptimal solutions.

\section{IP FORMULATION BY DISCRETIZATION OF THE SpaCe, Time AND CURVATURE}

In the following, we try to solve using discretization in the set $\Omega \in \mathfrak{R}^{2}$, curvature functions $\kappa_{i}$ and finally time.

\section{A. Discretization of $\Omega$ and time}

We partition the search space $\Omega$ into regions, using circles with radius $r$. The tessellation of the field to be observed is based on the geometry of the least capable camera and also the required resolution.

Let $B_{r}(p)$ represent the ball of radius $r$ and center $p$ as

$$
B_{r}(p)=\left\{q \in \mathfrak{R}^{2} \mid\|p-q\|_{2} \leq r\right\}
$$

The collection $\mathscr{C}=\left\{B_{r}\left(p_{1}\right), \ldots, B_{r}\left(p_{n}\right)\right\}$ is said to cover $\Omega$, or to be a covering of $\Omega$ if $\Omega \subseteq \bigcup_{i=1}^{n} B_{r}\left(p_{i}\right)$.

Given a covering $\mathscr{C}$, the discretized $\Omega$ or $\Omega^{d}$ is defined as the following set:

$$
\Omega^{d}=\left\{B_{r}(p) \in \mathscr{C} \mid B_{r}(p) \bigcap \Omega \neq \emptyset\right\}
$$

There exist methods for finding the minimum number of disks of radius $r$ to cover region $\Omega$ in the plane [20], however the number of the covering doesn't much effect the complexity of the coverage algorithm.

Similarly we define the discretized field of view $\psi_{i}^{d}\left(s_{i}(t)\right)$ as follows:

$$
\psi_{i}^{d}\left(s_{i}(t)\right)=\left\{B_{r}(p) \in \Omega^{d} \mid B_{r}(p) \subseteq \psi_{i}\left(s_{i}(t)\right)\right\}
$$

We also define discretized coverage map $\Psi^{d}(t)$ as :

$$
\Psi^{d}(s(t))=\bigcup_{i=1}^{N} \psi_{i}^{d}\left(s_{i}(t)\right)
$$

\section{B. Discretization of curvature $\kappa_{i}$}

Since the range of the function $\kappa_{i}:\left[t_{0}, t_{1}\right] \rightarrow\left[-1 / \rho_{i}, 1 / \rho_{i}\right]$ is bounded, we can fairly approximate function $\kappa_{i}(t)$ with piecewise constant function $\kappa_{i}^{d}(t)$ as:

$$
\kappa_{i}^{d}:\left[t_{0}, t_{1}\right] \rightarrow K(\rho)
$$

Where

$$
K(\rho)=\left\{\kappa_{i} \mid \kappa_{i}=\frac{i}{m \rho}, i=-m,-m+1, \ldots, m\right\}
$$

Also we assume that curvature is constant for a time interval with length $\delta_{1}$. In order to make $\kappa_{i}^{d}(t)$ a continuous function, we assume that the change of curvature between two different values $k_{j}$ to $k_{l}$ is linear in time and takes $\delta_{2}$ sec. The transition between $k_{j}$ to $k_{l}$ is possible iff $|l-j| \leq 1$.

\section{Integer Programming Formulation}

This section presents a Integer Programming (IP) formulation for the time critical cooperative coverage problem using discretized curvature and discretized field of view.

To proceed, assume that time is discretized. At any time (stage) $\mathrm{t}$, the decision (control) $\kappa(i)=\left(\kappa_{1}^{d}\left(t_{i}\right), \ldots, \kappa_{N}^{d}\left(t_{i}\right)\right)$ is defined to be a vector of constant curvatures for $\mathrm{N}$-vehicle system. Also lets assume that no-fly zones can be represented as the union of the regions $A_{k} \gamma<B_{k}$. The coverage problem 
can be stated as the following integer programming (IP) formulation:

$$
\begin{gathered}
\arg \min _{\{\kappa(1), \kappa(2), \cdots, \kappa(s)\}}\left[\operatorname{area}\left(\Omega-\bigcup_{i, t} \Psi_{i}\left(\gamma_{i}(t)\right)\right)\right] \\
\gamma_{i}\left(t_{i}^{\text {entry }}\right)=\gamma_{i}^{\text {entry }} \\
\left\|\gamma_{i}\left(t_{i}^{o}\right)-\gamma_{i}^{\text {exit }}\right\| \leq \alpha_{i}^{d} \\
\left|t_{i}^{o}-t_{i}^{\text {exit }}\right| \leq \alpha_{i}^{t} \\
\left\|\gamma_{i}(t)-\gamma_{j}(t)\right\|>d_{\text {safe }} \\
A_{k} \gamma_{i}(t)>B_{k}
\end{gathered}
$$

Where the first three constraints are boundary conditions and the forth and fifth constraints are collision avoidance an no-fly zone respectively. Since the size of resulting IP is very large in the following we try to break down the size of the IP by solving sequence of smaller size IP's using receding horizon control (RHC) approach. Now lets consider the RHC formulation for (23). Since in RHC method the planning horizon is usually much smaller than total mission time, therefore we can't enforce directly the final condition constraint $\left|t_{i}^{o}-t_{i}^{\text {exit }}\right| \leq \alpha_{i}^{t}$ in the optimization constraints. Hence we try to satisfy $\left|t_{i}^{o}-t_{i}^{\text {exit }}\right| \leq \alpha_{i}^{t}$ through the the terminal cost. Assume that the planning horizons for the RHC are $[i \tau,(i+1) \tau] \quad i=m, m+1, \cdots, n$ where $m \tau=t^{\text {entry }}$ and $n \tau=t^{\text {exit }}$, Now the RHC for the time period $[k \tau,(k+1) \tau)]$ is given by:

$$
\begin{gathered}
\arg \min \left[\operatorname{area}\left(\Omega(k-1)-\bigcup_{i} \psi_{i}\left(\gamma_{i}(t)\right)\right)\right]+ \\
\sum_{i} C\left(\gamma_{i}((k+1) \tau), \gamma_{i}^{\text {exit }}, \gamma_{i}^{\text {exit }}\right) \\
\gamma_{i}(k \tau)=\gamma_{i}^{\text {exit }}((k-1) \tau) \\
\left\|\gamma_{i}(t)-\gamma_{j}(t)\right\|>d_{\text {safe }} \\
A_{k} \gamma^{i}(t)>B_{k}
\end{gathered}
$$

Where the $\Omega(k-1)$ is the area which has not been covered yet and we have $\Omega(m)=\Omega$. Also $C\left(\gamma_{i}((k+1) \tau), \gamma_{i}^{\text {exit }}, t_{i}^{\text {exit }}\right)$ is the terminal cost for the vehicle $i$

1) Selecting the terminal cost: Assume that the for any state of the vehicle $s(i)$ the minimum time trajectory from $s(i)$ to the goal $s_{i}^{\text {exit }}$ can be approximately computed using visibility graph and the Dijkstra's graph shortest path algorithm [25]. Also assume that the min time of traveling is given by $T_{i}\left(\gamma_{i}(t), \gamma_{i}^{\text {exit }}\right)$. We define the terminal cost function $C($.$) as follows:$

$$
C\left(\gamma_{i}(t), \gamma_{i}^{\text {exit }}, t_{i}^{\text {exit }}\right)=
$$

$$
\left\{\begin{array}{c}
0 \text { if }\left(t_{i}^{\text {exit }}-\left(t+T_{i}\left(\gamma_{i}(t), \gamma_{i}^{\text {exit }}\right)\right)\right)>t_{\text {safe }} \\
\frac{1}{t_{i}^{\text {exit }}-\left(t+T_{i}\left(\gamma_{i}(t), \gamma_{i}^{\text {exit }}\right)\right)+\varepsilon} \text { if } 0 \leq t_{i}^{\text {exit }}-\left(t+T_{i}\left(\gamma_{i}(t), \gamma_{i}^{\text {exit }}\right)\right)<t_{\text {safe }} \\
\infty \text { if }\left(t_{i}^{\text {exit }}-\left(t+T_{i}\left(x_{i}(t), \gamma_{i}^{\text {exit }}\right)\right)\right)<0
\end{array}\right.
$$

So basically, the cost function looks at the min time collision free trajectory to the final state $\gamma_{i}^{e x i t}$ and compares it with the time that is left for the vehicle, if the vehicle has enough time the cost is set to zero and the main objective is to cover as much area as it can. As the remaining time shrinks the priority changes from coverage to just simply going directly to the target. One sample scenario is shown in figure 3.

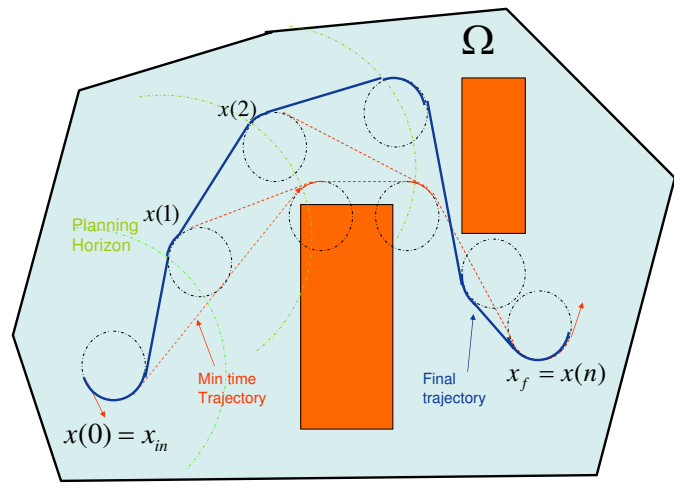

Fig. 3. Sample execution of receding horizon based method

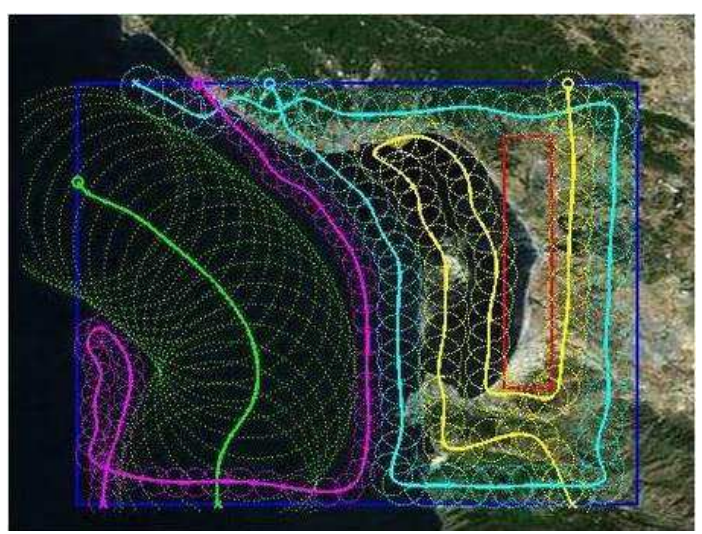

Fig. 4. RHC based solution with 61 min run time and $90 \%$ coverage

\section{Simulations}

\section{A. Problem scenario}

We test the proposed algorithms on the following scenario in

In the scenario the overage region is a rectangular $400 \times$ $300 \mathrm{~km}$ region (blue rectangle) centered at the origin with a rectangular $34 \times 179 \mathrm{~km}$ no fly-sail zone (red rectangle). we consider a fleet of four heterogeneous vehicles with different speeds, sensors,task durations, entry and exit states.

- Two UAVs (yellow and cyan) with constant speeds $111 \mathrm{~m} / \mathrm{sec}$, each equipped with camera which has a $15 \mathrm{~km}$ radius footprint and task durations $129 \mathrm{~min}, 165 \mathrm{~min}$ respectively. The entry points are $p_{1}^{\text {entry }}=(153,-150), p_{2}^{\text {entry }}=(-159,150)$ and exit points are $p_{1}^{\text {exit }}=(150,150), p_{2}^{\text {exit }}=(-63,150)$.

- One Unmanned Surface Vehicle (USV); color green in the Fig. ??; with constant speed $11 \mathrm{~m} / \mathrm{sec}$, equipped with radar with range of $70 \mathrm{~km}$ radius and $445 \mathrm{~min}$ task duration. The entry and exit points are $p_{3}^{\text {entry }}=$ $(-100,-150), p_{3}^{\text {exit }}=(-200,80)$.

- One Tactical UAV; color magenta in the Fig. ??; with constant speed $55 \mathrm{~m} / \mathrm{sec}$ equipped with camera which has a $10 \mathrm{~km}$ radius footprint and $230 \mathrm{~min}$ task duration. The entry and exit points are $p_{4}^{\text {entry }}=(-182,-150)$, $p_{4}^{\text {exit }}=(-114,150)$. 


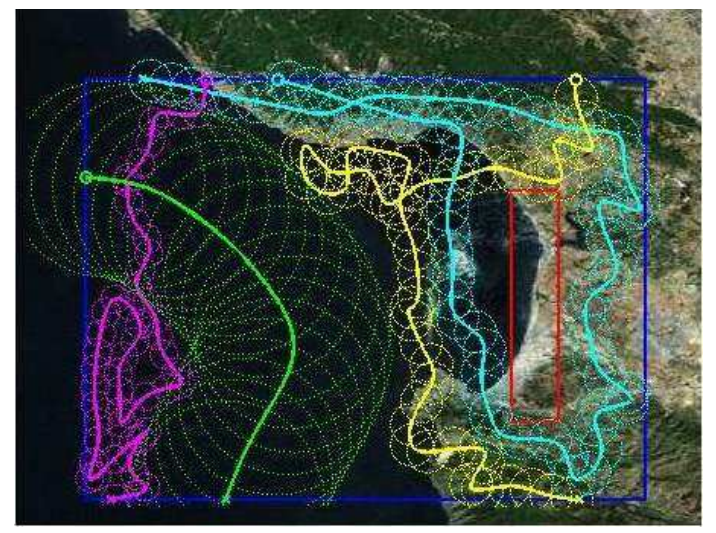

Fig. 5. RHC based solution with 83 sec. run time and $83 \%$ coverage

\section{B. Computed results}

We used the receding horizon algorithm to compute a solution for the problem in Fig. 4. The solution is close to optimal (around 90\% coverage). However, the computational time is about 1 hour, which is far more than the given time budget. In RHC algorithm we can decrease computation time by reducing planning horizon and with the price of reduction in the optimality of the solution. Fig. 5 shows a solution to the coverage problem with $80 \%$ coverage and 83 seconds running time.

\section{CONCLUSION}

In this paper we presented a path planning algorithm for time sensitive cooperative surveillance using UAVs equipped with cameras. There are two additional avenues for further research. First, the results in this paper addressed twodimensional sensor footprints and coverage problems. There is a need to combine data from UGVs and UAVs, or UUVs and USVs with UAVs which require the extension to threedimensional settings. Second, as the number of vehicles increase, it will become necessary to solve the problem using decentralized algorithms. This is also an area of great interest.

\section{REFERENCES}

[1] S. Bayraktar, G. Fainekos and G. Pappas, Experimental Cooperative Control of Fixed-Wing Unmanned Aerial Vehicles, In the Proceedings of the 43rd IEEE Conference on Decision and Control, The Bahamas, December 2004

[2] V. Gavrilets, A. Shterenberg, M. Dehaleh and E. Feron, Avionics System for a Small Unmanned Helicopter Performing Aggressive Maneuvers, in 19th Digital Avionics Systems Conference Proceedings, 7-13 Oct. 2000.

[3] H.J. Kim, D.H. Shim, S. Sastry, Flying robots: modeling, control and decision making, in Proceedings of International Conference on Robotics and Automation, ICRA 02, 11-15 May 2002

[4] E. Olsen, C.W. Park, and J. How, 3D Formation Flight using Differential Carrier-phase GPS Sensors, The J. of The Institute Of Navigation, Spring, 1999, Vol. 146, No. 1, pp. 3548.

[5] J. Evans, G. Inalhan, J.S. Jang, R. Teo, C.J. Tomlin, DragonFly: a versatile UAV platform for the advancement of aircraft navigation and control, in 20th Conference Digital Avionics Systems, 14-18 Oct. 2001

[6] Fierro, R., Belta, C., Desai, J.P. and Kumar, V., On Controlling Aircraft Formations, Proceedings of the 40th IEEE Conference on Decision and Control, Orlando, FL, Dec. 2001, vol. 2, pp. 1065-70.
[7] L. E. Dubins, On curves of minimal length with a constraint on average curvature, and with prescribed initial and terminal positions and tangents, Amer. J. Math., vol. 79, no. 3, pp. 497-516, 1957

[8] K. Savla, E. Frazzoli, and F. Bullo. On the point-to-point and traveling salesperson problems for Dubins' vehicles. In proceedings of the American Control Conference, 2005.

[9] S. Rathinam, R. Sengupta, A Safe Flight Algorithm for Unmanned Aerial Vehicles, in IEEE Aerospace Conference, Montana, 2004

[10] S. Rathinam, M. Zennaro, T. Mak and R. Sengupta, An architecture for UAV team control, Proc. IFAC Conference on Intelligent Autonomous Vehicles in Portugal, July 2004.

[11] A. Ahmadzadeh, B. Sayyar-Roudsari and A. Homaifar, A Hybrid Evolutionary-Gradient Search Algorithm for Capacitated Multi-Source Multi-UAVs Scheduling with Time Windows, Recent Developments in Cooperative Control and Optimization Edt. S. Butenko, R. Murphey, and M. Pardalos, Kluwer Academic Publishers, December 2003

[12] A. Richards, J. How, Mixed-integer Programming for Control, In proceedings of the American Control Conference, June 2005.

[13] H. Choset, Coverage for robotics-a survey of recent results, Annals of Mathematics and Artificial Intelligence Vol. 31, pp. 113-126, 2001

[14] D. A. Castanon, Approximated dynamic programming for sensor management, in Proc. IEEE Conf. Decision Control, San Diego, CA, pp. 1202-1207. Dec. 1997

[15] D. J. Cool, P. Gmytrasiewicz, and L. B. Holder, Decision-theoretic cooperative sensor planning, IEEE Trans. Pattern Anal. Machine Intell., vol. 18, no. 10, pp. 892-902, Oct. 1996.

[16] M. Kalandros, L. Y. Pao, and Y. Ho, "Randomization and superheuristics in choosing sensor sets for target tracking applications, in Proc. IEEE Conf. Decision Control, vol. 2, Phoenix, AZ, pp. 18031808. Dec. 1999

[17] P. Vanheeghe, E. Duflos, P. E. Dumont, and V. Nimier, Sensor management with respect to danger level of targets, in Proc. IEEE Conf. Decision Control, vol. 5, Orlando, FL, pp. 4439-4444, 2001

[18] J. Cortes, S. Martinez, T. Karatas, and F. Bullo, Coverage control for mobile sensing networks. In Proceedings of IEEE International Conference on Robotics and Automation, 2002, pp. 1327-1332.

[19] J.J. Enright and E. Frazzoli and K. Savla and F. Bullo. On Multiple UAV Routing with Stochastic Targets: Performance Bounds and Algorithms. Proc. of the AIAA Conf. on Guidance, Navigation, and Control, August 2005.

[20] Do Carmo M.P., Differential geometry of curvs and surfaces, Prentice Hall, 1976.

[21] A. Ahmadzadeh, J. Keller, G. Pappas, A. Jadbabaie and V. Kumar, Multi-UAV Deployment for Coverage with Spatio-Temporal Specifications IEEE Conference on Decision and Control 2006

[22] A. Ahmadzadeh, J. Keller, G. Pappas, A. Jadbabaie and V. Kumar, An Optimization-based Approach to Time Critical Cooperative Surveillance and Coverage with Unmanned Aerial Vehicles, In International Symposium on Experimental Robotics 2006

[23] R. Kershner, The number of circles covering a set. Amer. J. Math., vol. 61, pp. 665-671, 1939.

[24] Jadbabaie, A., Yu, J., and Hauser, J., Unconstrained receding-horizon control of nonlinear systems,IEEE Transactions on Automatic Control, Vol. 46, No. 5, May 2001, pp. 776-783.

[25] J. Bellingham, A. Richards, and J. How, Receding Horizon Control of Autonomous Aerial Vehicles, in Proceedings of the American Control Conference, May 2002. 